\title{
Energy Harvesting with Piezoelectric Element Using Vibroacoustic Coupling Phenomenon
}

\author{
Hiroyuki Moriyama, ${ }^{1}$ Hirotarou Tsuchiya, ${ }^{2}$ and Yasuo Oshinoya ${ }^{1}$ \\ ${ }^{1}$ Department of Prime Mover Engineering, Tokai University, 4-1-1 Kitakaname, Hiratsuka, Kanagawa 259-1292, Japan \\ ${ }^{2}$ Rolling Stock Drive Systems Department, Toshiba Transport Engineering Inc. 1 Toshiba-cho, Fuchu, Tokyo 183-8511, Japan \\ Correspondence should be addressed to Hiroyuki Moriyama; moriyama@keyaki.cc.u-tokai.ac.jp
}

Received 3 October 2013; Accepted 31 October 2013

Academic Editor: Abdelkrim Khelif

Copyright (c) 2013 Hiroyuki Moriyama et al. This is an open access article distributed under the Creative Commons Attribution License, which permits unrestricted use, distribution, and reproduction in any medium, provided the original work is properly cited.

\begin{abstract}
This paper describes the vibroacoustic coupling between the structural vibrations and internal sound fields of thin structures. In this study, a cylindrical structure with thin end plates is subjected to the harmonic point force at one end plate or both end plates, and a natural frequency of the end plates is selected as the forcing frequency. The resulting vibroacoustic coupling is then analyzed theoretically and experimentally by considering the dynamic behavior of the plates and the acoustic characteristics of the internal sound field as a function of the cylinder length. The length and phase difference between the plate vibrations, which maximize the sound pressure level inside the cavity, are clarified theoretically. The theoretical results are validated experimentally through an excitation experiment using an experimental apparatus that emulates the analytical model. Moreover, the electricity generation experiment verifies that sufficient vibroacoustic coupling can be created for the adopted electricity generating system to be effective as an electric energy-harvesting device.
\end{abstract}

\section{Introduction}

Recently, scavenging ambient vibration energy and converting it into usable electric energy via piezoelectric materials have attracted considerable attention [1]. Typical energy harvesters adopt a simple cantilever configuration to generate electric energy via piezoelectric materials, which are attached to or embedded in vibrational elements. High-amplitude excitations reduce the fatigue life of these harvesters. Thus, placing appropriate constraints on the amplitudes is one of significant ways to improve the performance of harvesters. A cantilever beam, whose deflection was constrained by a bump stop, was modeled. The effect of electromechanical coupling was estimated in a parametric study, where the placement of the bump stop and the gap between the beam and stop were chosen as parameters [2]. Acoustic energy as well as vibration energy to be harvested sufficiently fills our working environment. Thermoacoustic engines that exploit the inherently efficient Stirling cycle and are designed on the basis of a simple acoustic apparatus with no moving parts have been regarded as the representative means for harvesting acoustic energy [3]. As an example application, electricity generation using resonance phenomena in a thermoacoustic engine was investigated with aim of harvesting the work done in the engine. The acoustic energy spent on electricity generation was harvested from a resonance tube branching out of the engine, and the appropriate position of the resonance tube for effectively generating electricity was described [4]. Moreover, an electricity generating device using the sound of voice via piezoelectric elements was developed as a supplementary electric source for a mobile phone [5]. This system is also being investigated for other applications [6]. Acoustic energy is extremely small in comparison to vibration energy. However, the above-mentioned electricity is generated by sound and vibration. Therefore, vibroacoustic coupling is one way to increase acoustic energy, although it has not yet been investigated extensively in this context.

Vibroacoustic coupling was investigated as an architectural acoustic problem via a coupled panel-cavity system consisting of a rectangular box with slightly absorbing walls and 
a simply supported panel. The effect of the panel characteristics on the decay behavior of the sound field in the cavity was considered both theoretically and experimentally $[7,8]$. In an attempt to control noise in an airplane, an analytical model for investigating coupling between the sound field in an aircraft cabin and the vibrations of the rear pressure bulkhead was proposed $[9,10]$. A cylindrical structure adopted as the analytical model, in which the rear pressure bulkhead at one end of the cylinder was assumed to be a circular plate, was examined under various conditions. The plate was supported at its edges by springs whose stiffness could be adjusted to simulate the various support conditions. These investigations clarified the influence of the support conditions on the sound pressure of an internal sound field coupled with the vibration of the end plate. The authors of this study used the abovementioned analytical model $[9,10]$ of a cylindrical structure with plates at both ends to investigate the vibroacoustic coupling based on the sound pressure level distribution in the cavity. The acoustic characteristics under vibroacoustic coupling were investigated for cases in which excitation forces with different relative amplitudes and phases were applied to both end plates [11]. In addition, the excitation frequency at which the coupling system becomes nonperiodic owing to the application of excitation forces of different frequencies to the respective end plates was investigated [12]. Finally, the effect of the excitation position with respect to the nodal lines on the appearance of vibration modes on the plates was investigated [13]. On the other hand, to suppress the above vibration and acoustic energy, which was amplified by vibroacoustic coupling, an analytical model that included the installation of passive devices on the vibration system was proposed as the electromechanical-acoustic system. The effect was fully validated in the numerical approach owing to tuning the resonance characteristics of the shunt circuit, in which the piezoelectric device was incorporated, to the frequency characteristics of the coupling system [14].

In almost all such studies, vibroacoustic coupling has been estimated by assuming that the plate and cavity dimensions, as well as the phase difference between the vibroacoustics of the two plates, were fixed. However, the natural frequencies of the plate and cavity vary with the dimensions of the plate and cavity, and the phase difference directly affects the sound field when the medium is much less dense than the plate. Although the study of coupling phenomena had attracted attention, an extensive parametric study has not yet been undertaken. Hence, little is known about the influence of dimensions and phase differences on the coupling phenomena.

To develop a new electricity generation system, we adopt an analytical model similar to the above-mentioned cylindrical structure with plates at both ends, because the vibration area of the model on which piezoelectric elements can be installed is twice as large as that in case of a single plate. The cylinder length is varied over a wide range, while the harmonic point force is applied to one end plate or both end plates, and its frequency is selected to cause the plate to vibrate in the fundamental mode. Vibroacoustic coupling that occurs between plate vibrations and the sound field in the cavity is investigated theoretically and experimentally in

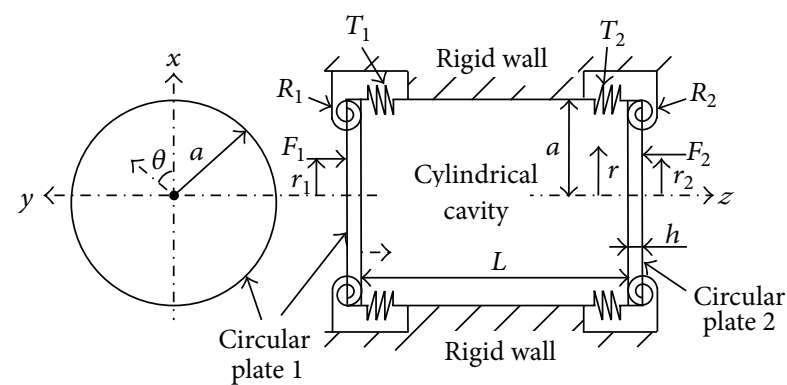

FIgURE 1: Configuration of analytical model.

terms of the vibration and acoustic characteristics. In the experiment, the acceleration of the plate vibrations, the phase difference between them, and the sound pressure level inside the cavity are considered significant characteristics of the plate vibrations and sound field. These experimental results demonstrate the underlying theoretical analysis based on this model, as well as the conditions that maximize the vibration and sound pressure levels. Furthermore, the effect of vibroacoustic coupling is estimated from an electricity generation experiment performed with piezoelectric elements.

\section{Analytical Method}

2.1. Equation of Motion of Plate. The analytical model consists of a cavity with two circular end plates, as shown in Figure 1. The plates are supported by translational and rotational springs distributed at constant intervals, and the support conditions are determined by their respective spring stiffness $T_{1}, T_{2}, R_{1}$, and $R_{2}$, where the suffixes 1 and 2 indicate plates 1 and 2 , respectively. The plates of radius $a$ and thickness $h$ have a Young's modulus $E$ and a Poisson's ratio $\nu$. The sound field, assumed cylindrical, has the same radius as that of the plates and varying length because the resonance frequency depends on the length. The boundary conditions are considered structurally and acoustically rigid at the lateral wall between the structure and sound field. The coordinates used are radius $r$ and angle $\theta$ between the planes of the plates and the cross-sectional plane of the cavity and distance $z$ along the cylinder axis. The periodic point forces $F_{1}$ and $F_{2}$ are applied to plates 1 and 2 at distances $r_{1}$ and $r_{2}$ and angles $\theta_{1}$ and $\theta_{2}$, respectively. The natural frequency of the plates is employed as the excitation frequency.

To formulate the plate motion, Hamilton's principle is applied to the analytical model [9]:

$$
\delta H=\delta \int_{t_{0}}^{t_{1}}\left(T_{P}-U_{P}-U_{S}+W\right) d t=0,
$$

where $H$ is the Hamiltonian, $T_{P}$ and $U_{P}$ are, respectively, the kinetic and potential energy of each plate, and $U_{S}$ is the elastic energy stored in the springs. $W$ is the work done on plates 1 and 2 by the respective point forces and the sound pressure on the plates. Finally, $t_{0}$ and $t_{1}$ are two arbitrary times. 
The flexural displacements $w_{1}$ and $w_{2}$ of plates 1 and 2, respectively, in terms of two sets of suitable trial functions are found by substituting (3) for the plate modes into (2) below:

$$
\begin{gathered}
w_{1}=\sum_{s=0}^{1} \sum_{n=0}^{\infty} \sum_{m=0}^{\infty} B_{1 n m}^{s} X_{n m}^{s} e^{j\left(\omega t+\phi_{1}\right)}, \\
w_{2}=\sum_{s=0}^{1} \sum_{n=0}^{\infty} \sum_{m=0}^{\infty} B_{2 n m}^{s} X_{n m}^{s} e^{j\left(\omega t+\phi_{2}\right)}, \\
X_{n m}^{s}=\sin \left(n \theta+\frac{s \pi}{2}\right)\left(\frac{r}{a}\right)^{m},
\end{gathered}
$$

where $n, m$, and $s$ are, respectively, the circumferential order, radial order, and symmetry index with respect to the plate vibration. $B_{1 n m}^{s}$ and $B_{2 n m}^{s}$ are coefficients to be determined, $\omega$ is the angular frequency of the harmonic point force acting on the plate, and $t$ is the elapsed time. $\phi_{1}$ and $\phi_{2}$ are the phases of the respective plate vibrations. In this analysis, $\phi_{1}$ is set to $0 \mathrm{deg}$, and $\phi_{2}$ varies in the range of $0 \mathrm{deg}$ to $180 \mathrm{deg}$. $w_{1}$ and $w_{2}$ are substituted for the flexural displacements in $T_{P}, U_{P}, U_{S}$, and $W$, whose detailed expressions are obtained from $[9,10]$, and the variation of (1) is carried out with respect to both plates. Consequently, the extremum of the Hamiltonian yields Euler's equations, which are the equations of motion of the respective plates. The motion is assumed to be harmonic, that is, to behave as $e^{j \omega t}$, so that $e^{j \omega t}$ can be eliminated.

Consider the following:

$$
\begin{aligned}
& {\left[\sum_{m^{\prime}=0}^{\infty}\left\{K_{1 n m m^{\prime}}^{s}\left(1+j \eta_{p}\right)-\omega^{2} M_{1 n m m^{\prime}}^{s}\right\}\right.} \\
& \left.+\sum_{m^{\prime}=0}^{\infty} a F_{s n}\left\{T_{1}+\left(\frac{m}{a}\right)\left(\frac{m^{\prime}}{a}\right) R_{1}\right\}\right] \mathbf{B}_{1 n m^{\prime}}^{s} e^{j \phi_{1}} \\
& =\mathbf{F}_{1 n m}^{s}-\mathbf{P}_{1 n m}^{s}, \\
& {\left[\sum_{m^{\prime}=0}^{\infty}\left\{K_{2 n m m^{\prime}}^{s}\left(1+j \eta_{p}\right)-\omega^{2} M_{2 n m m^{\prime}}^{s}\right\}\right.} \\
& \left.+\sum_{m^{\prime}=0}^{\infty} a F_{s n}\left\{T_{2}+\left(\frac{m}{a}\right)\left(\frac{m^{\prime}}{a}\right) R_{2}\right\}\right] \mathbf{B}_{2 n m^{\prime}}^{s} e^{j \phi_{2}} \\
& \quad=-\mathbf{F}_{2 n m}^{s}+\mathbf{P}_{2 n m}^{s},
\end{aligned}
$$

where $K_{1 n m m^{\prime}}^{s}, K_{2 n m m^{\prime}}^{s}$ and $M_{1 n m m^{\prime}}^{s}, M_{2 n m m^{\prime}}^{s}$ are elements of the symmetrical stiffness and mass matrices, respectively, because the index $m^{\prime}$ is the radial order $\left(m=m^{\prime}\right) \cdot \eta_{p}$ is the structural damping factor of the plate, and $F_{s n}$ is a coefficient that is determined by the indices $n$ and $s$ and is expressed as [9]

$$
F_{s n}= \begin{cases}\pi, & \text { at } n \neq 0, \\ 0, & \text { at } n=0, s=0, \\ 2 \pi, & \text { at } n=0, s=1\end{cases}
$$

Both first terms on the right-hand sides of (4) give the respective point forces, and the second terms give the acoustic excitations, which also function as the coupling term between each plate vibration and the sound field. The point force and acoustic excitation terms are, respectively,

$$
\begin{gathered}
F_{1 n m}^{s}=\int_{A_{1}} F_{1} \delta\left(r-r_{1}\right) \delta\left(\theta-\theta_{1}\right) X_{n m}^{s} d A_{1}, \\
F_{2 n m}^{s}=\int_{A_{2}} F_{2} \delta\left(r-r_{2}\right) \delta\left(\theta-\theta_{2}\right) X_{n m}^{s} d A_{2}, \\
P_{1 n m}^{s}=\int_{A_{1}} P_{c} X_{n m}^{s} d A_{1}, \quad P_{2 n m}^{s}=\int_{A_{2}} P_{c} X_{n m}^{s} d A_{2} .
\end{gathered}
$$

Here $\delta$ is the delta function associated with the point force on plates, $A_{1}$ and $A_{2}$ are the plate areas, and $P_{c}$ is the sound pressure at an arbitrary point on the boundary surface of the plates. To distinguish between plates 1 and 2, the differential $d A(r d \theta d r)$ is written as $d A_{1}$ and $d A_{2}$ in (6) and (7), respectively.

\subsection{Coupling Equation between Plate Vibrations and Internal} Sound Field. For simplicity, we assume that the cavity walls are rigid, so that the sound field in the cavity is governed by the wave equation consisting of the eigenfunction $Y_{N}$ and the eigenvalue $k_{N}$ corresponding to a cavity mode of order $N$ :

$$
\begin{gathered}
\nabla^{2} Y_{N}+k_{N}^{2} Y_{N}=0, \\
\left(\frac{\partial Y_{N}}{\partial \mathbf{u}}\right)_{S}=0
\end{gathered}
$$

where $\mathbf{u}$ is the unit normal to the boundary surface $S$ (positive towards the outside), and the boundary condition satisfies (9) when $S$ is rigid. However, if $S$ is not rigid but has a varying specific acoustic admittance, we select a Green's function $G$ to obtain a solution set for a nonuniform cavity with nonrigid walls for a frequency $\omega / 2 \pi=K c / 2 \pi$, where $K$ is an eigenvalue of the nonuniform cavity, and $c$ is the cavity speed of sound. The equation for $G$ is thus given by

$$
\begin{aligned}
\nabla^{2} G+K^{2} G= & -\delta\left(\mathbf{p}-\mathbf{p}_{0}\right) \\
= & -\delta\left(r-r_{0}\right) \delta\left(\theta-\theta_{0}\right) \delta\left(z-z_{0}\right), \\
& \left(\frac{\partial G}{\partial \mathbf{u}}\right)_{S}=0 .
\end{aligned}
$$

The right-hand side is a delta function, where the measurement point is $\mathbf{p}=(r, \theta, z)$ if the source point is $\mathbf{p}_{0}=$ $\left(r_{0}, \theta_{0}, z_{0}\right)$. Expressing $G$ in terms of $Y_{N}$ of (8), which satisfies the same boundary conditions, we find that

$$
\begin{gathered}
G\left(\mathbf{p}, \mathbf{p}_{0}\right)=\sum_{N=1}^{\infty} \frac{Y_{N}(\mathbf{p}) Y_{N}\left(\mathbf{p}_{0}\right)}{V_{c} M_{N}\left(k_{N}^{2}-K^{2}\right)}, \\
\int_{V_{c}} Y_{N}(\mathbf{p}) Y_{M}(\mathbf{p}) d V_{c}=V_{c} M_{N} \delta_{N M}= \begin{cases}0 & \text { at } N \neq M, \\
V_{c} M_{N} & \text { at } N=M .\end{cases}
\end{gathered}
$$

The dimensionless factor $M_{N}$ is the mean value of $Y_{N}^{2}$ averaged over the cavity volume $V_{c}$, and $\delta_{N M}$ is the Kronecker delta.

Because there is no source and $\partial G / \partial \mathbf{u}=0$ on $S$, the spatial factor $P_{c}(\mathbf{p})$ of the sound pressure within and on the surface 
bounding the medium can be obtained from just one of the surface integral terms as follows:

$$
P_{c}(\mathbf{p})=-\int_{S} G\left(\mathbf{p}, \mathbf{p}_{0}\right) \frac{\partial P_{c}\left(\mathbf{p}_{0}\right)}{\partial \mathbf{u}_{0}} d S_{0},
$$

where the zero subscripts indicate differentiation and integration with respect to the $\left(r_{0}, \theta_{0}, z_{0}\right)$ coordinates. A detailed procedure for obtaining these equations is given in [15]. $P_{c}$ can also be expressed as $[9,10]$

$$
P_{c}=\rho_{c} c^{2} \sum_{N=1}^{\infty} \frac{P_{N} Y_{N}}{M_{N}}
$$

where $\rho_{c}$ is the fluid density in the cavity, and $P_{N}$ is the pressure coefficient to be determined.

In this investigation, the acoustic modal shape $Y_{n p q}^{s}$ and angular resonance frequency $\omega_{n p q}$ in the cavity (where the indices $n, p$, and $q$ indicate the circumferential, radial and longitudinal orders, resp.) are defined as

$$
\begin{gathered}
Y_{n p q}^{s}=\sin \left(n \theta+\frac{s \pi}{2}\right) J_{n}\left(\lambda_{n p} r\right) \cos \left\{\left(\frac{q \pi}{L}\right) z\right\}, \\
\omega_{n p q}=c\left\{\lambda_{n p}^{2}+\left(\frac{q \pi}{L}\right)^{2}\right\}^{1 / 2},
\end{gathered}
$$

where $J_{n}$ is the $n$ th-order Bessel function, and $\lambda_{n p}$ is the $p$ th solution of an eigenvalue problem for a circular sound field having modes $(n, p)$ divided by the radius. The boundary conditions between the plate vibrations and sound field on the respective plate surfaces are found by assuming continuity of the velocities on the plates:

$$
\left(\frac{\partial P_{c}}{\partial \mathbf{u}}\right)_{z=0}=\rho_{c} \omega^{2} w_{1}, \quad\left(\frac{\partial P_{c}}{\partial \mathbf{u}}\right)_{z=L}=-\rho_{c} \omega^{2} w_{2},
$$

where $\partial P_{c} / \partial \mathbf{u}$ is 0 on the lateral wall of the cylinder since the wall remains rigid.

Because the analytical mode has two boundary surfaces, we can apply (16) to (13), so that $P_{c}$ becomes

$$
P_{c}=-\int_{A_{1}} G \rho_{c} \omega^{2} w_{1} d A_{1}+\int_{A_{2}} G \rho_{c} \omega^{2} w_{2} d A_{2} .
$$

On the other hand, by substituting acoustic modes of three orders, $n, p$, and $q$, instead of the order $N$ of the cavity mode into (14), $P_{c}$ can also be expressed as

$$
P_{c}=\rho_{c} c^{2} \sum_{p=1}^{\infty} \sum_{q=0}^{\infty} \frac{P_{n p q}^{s} Y_{n p q}^{s}}{M_{n p q}^{s}} .
$$

The equation relating (17) and (18) is obtained by applying Green's function of (11) to an arbitrary acoustic mode $(n, p, q)$ as

$$
\begin{aligned}
& \left(\omega_{n p q}^{2}-\omega^{2}\right) P_{n p q}^{s} \\
& \quad=-\frac{\omega^{2}}{V_{c}}\left(\int_{A_{1}} Y_{n p q}^{s} w_{1} d A_{1}+\int_{A_{2}} Y_{n p q}^{s} w_{2} d A_{2}\right) .
\end{aligned}
$$

Here, substituting (2) for $w_{1}$ and $w_{2}$ and considering a modal damping factor $\eta_{c}$, (19) can be rewritten as

$$
\begin{gathered}
\left(\omega_{n p q}^{2}+j \eta_{c} \omega_{n p q} \omega-\omega^{2}\right) P_{n p q}^{s} \\
=\frac{A \omega^{2}}{V_{c}}\left(-\sum_{m=0}^{\infty} I_{1} B_{1 n m}^{s} e^{j \phi_{1}}+\sum_{m=0}^{\infty} I_{2} B_{2 n m}^{s} e^{j \phi_{2}}\right), \\
I_{1}=\frac{1}{A} \int_{A_{1}} X_{n m}^{s} Y_{n p q}^{s} d A_{1}, \quad I_{2}=\frac{1}{A} \int_{A_{2}} X_{n m}^{s} Y_{n p q}^{s} d A_{2},
\end{gathered}
$$

where $A$ is the total surface area of the plates, $I_{1}$ and $I_{2}$ are the spatial coupling coefficients. Moreover, substituting (18) for $P_{c}$ and applying $I_{1}$ and $I_{2}$ to the integrals in (7), the acoustic excitation terms $P_{1 \mathrm{~nm}}^{s}$ and $P_{2 n m}^{s}$ can be expressed with respect to an arbitrary vibration mode $(n, m)$ as

$$
\begin{aligned}
& P_{1 n m}^{s}=\rho_{c} c^{2} A \sum_{p=1}^{\infty} \sum_{q=0}^{\infty} \frac{I_{1} P_{n p q}^{s}}{M_{n p q}^{s}}, \\
& P_{2 n m}^{s}=\rho_{c} c^{2} A \sum_{p=1}^{\infty} \sum_{q=0}^{\infty} \frac{I_{2} P_{n p q}^{s}}{M_{n p q}^{s}} .
\end{aligned}
$$

Finally, replacing $P_{n p q}^{s}$ in (22) with those in (20) and then inserting them in (4), we can complete the coupling equations, whose right-hand sides are

$$
\begin{aligned}
\mathbf{F}_{1 n m}^{s}-\mathbf{P}_{1 n m}^{s}= & \mathbf{F}_{1 n m}^{s}+\frac{\rho_{c} c^{2} \omega^{2} A^{2}}{V_{c}} \\
& \times \sum_{m^{\prime}=0}^{\infty} \sum_{p=1}^{\infty} \sum_{q=0}^{\infty} \frac{I_{1}\left(I_{1} B_{1 n m^{\prime}}^{s} e^{j \phi_{1}}-I_{2} B_{2 n m^{\prime}}^{s} e^{j \phi_{2}}\right)}{M_{n p q}^{s}\left(\omega_{n p q}^{2}+j \eta_{c} \omega_{n p q} \omega-\omega^{2}\right)}, \\
-\mathbf{F}_{2 n m}^{s}-\mathbf{P}_{2 n m}^{s}= & -\mathbf{F}_{2 n m}^{s}+\frac{\rho_{c} c^{2} \omega^{2} A^{2}}{V_{c}} \\
& \times \sum_{m^{\prime}=0}^{\infty} \sum_{p=1}^{\infty} \sum_{q=0}^{\infty} \frac{I_{2}\left(I_{1} B_{1 n m^{\prime}}^{s} e^{j \phi_{1}}-I_{2} B_{2 n m^{\prime}}^{s} e^{j \phi_{2}}\right)}{M_{n p q}^{s}\left(\omega_{n p q}^{2}+j \eta_{c} \omega_{n p q} \omega-\omega^{2}\right)} .
\end{aligned}
$$

On the right-hand sides, the second terms show the acoustic excitation for plates 1 and 2, respectively. The acoustic excitation terms have both $I_{1}$ and $I_{2}$ since the acoustic mode of the sound field is coupled with the vibration modes of the respective plates. Before actual calculation, the natural frequency of the plate must be considered in terms of the convergence of the plate vibration mode $(n, m)$. In this case, the natural frequency is obtained as the eigenvalue of (4), whose right-hand side is set to 0 . The actual calculation is performed by taking 15 terms for $n$, while $m$ is set to be greater than 13 to ensure the convergence of the natural frequency and mode shape of the plate. Using the same truncation for $p$ as for $m$, the order accounts for acoustic modes greater than $q=15$, so that the resonance frequency containing $q$ can exceed the excitation frequency. The plate and cavity loss factors are assumed to be constant: $\eta_{p}=\eta_{c}=0.01[9,10]$. Since $B_{1 n m}^{s}$ and $B_{2 n m}^{s}$ can be obtained simultaneously from (4), which have as excitation terms, the behavior of the plate 


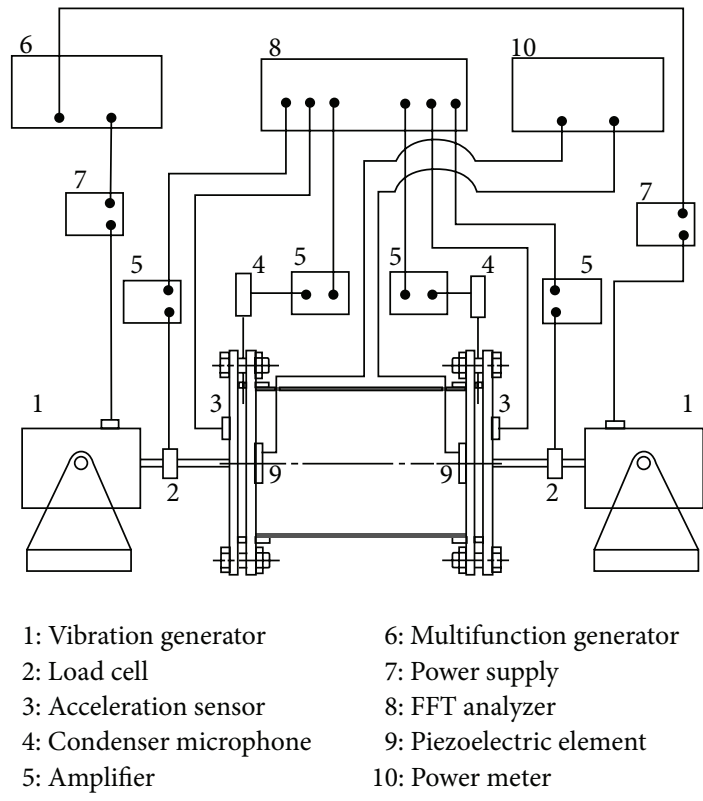

(a)

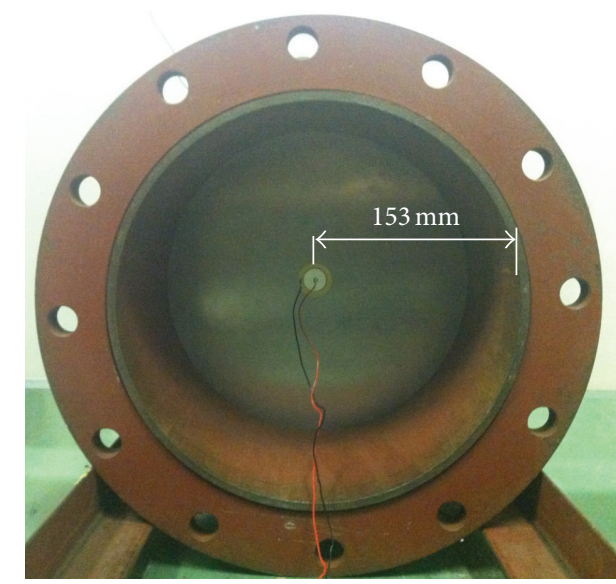

(b)

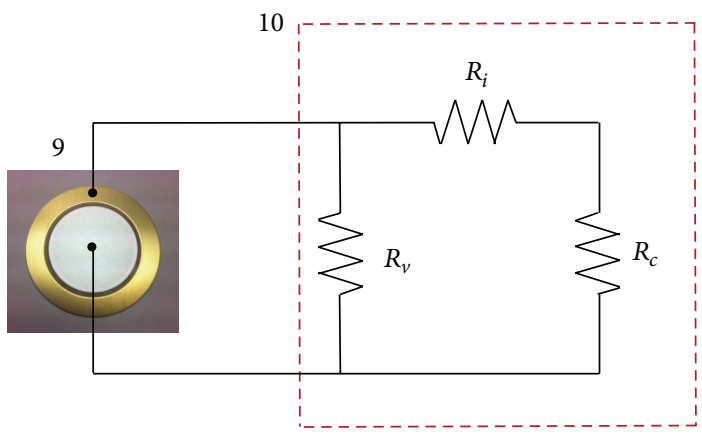

(c)

Figure 2: Configuration of experimental apparatus: (a) Measurement system; (b) Installation state of piezoelectric element; and (c) Electrical circuit of energy-harvesting device.

vibrations and the sound field under vibroacoustic coupling can be determined.

The flexural displacements $w_{1}$ and $w_{2}$ and the sound pressure $P_{c}$ are, respectively, obtained from (2) and (18) by employing $B_{1 n m}^{s}$ and $B_{2 n m}^{s}$ determined above. However, the procedure to calculate $P_{c}$ involves substituting $P_{n p q}^{s}$ in (20) for that in (18). Solved from (24), into which $w_{1}, w_{2}$, and $P_{c}$ are inserted, over the respective entire regions, the quadric velocities $V_{1}$ and $V_{2}$ and the sound pressure $P_{\nu}$ are estimated by the logarithmic values $L_{v 1}, L_{v 2}$, and $L_{p v}$ relative to $V_{0}=$ $2.5 \times 10^{-15} \mathrm{~m}^{2} / \mathrm{s}^{2}$ and $P_{0}=4 \times 10^{-10} \mathrm{~N}^{2} / \mathrm{m}^{4}$, respectively, as shown in (25).

Consider the following:

$$
\begin{aligned}
& V_{1}=\frac{\omega^{2}}{2 A_{1}} \int_{A_{1}} w_{1} w_{1}^{*} d A_{1}, \quad V_{2}=\frac{\omega^{2}}{2 A_{2}} \int_{A_{2}} w_{2} w_{2}^{*} d A_{2}, \\
& P_{v}=\frac{1}{2 V_{c}} \int_{V_{c}} P_{c} P_{c}^{*} d V_{c},
\end{aligned}
$$

$$
L_{v 1}=10 \log \frac{V_{1}}{V_{0}}, \quad L_{v 2}=10 \log \frac{V_{2}}{V_{0}}, \quad L_{p v}=10 \log \frac{P_{v}}{P_{0}},
$$

where $w_{1}^{*}, w_{2}^{*}$, and $P_{c}^{*}$ are the respective conjugate components.

\section{Experimental Apparatus and Method}

Figure 2(a) shows the experimental apparatus used in this study. The structure consists of a steel cylinder with circular aluminum end plates that are $3 \mathrm{~mm}$ thick. The cylinder has an inner radius of $153 \mathrm{~mm}$, and this length can be varied from 500 to $2000 \mathrm{~mm}$ to emulate the analytical model. One end plate or both end plates are subjected to the point force, whose frequency makes the plate excite in the $(0,0)$ mode. In case of the harmonic excitation of both ends, these forces are applied to the respective plates via small vibrators, and their amplitudes are controlled to be $1 \mathrm{~N}$. The positions of the point forces $r_{1}$ and $r_{2}$ are normalized by radius $a$ and 
are set to $r_{1} / a=r_{2} / a=0.4$. In the excitation experiment, the main characteristic is the phase difference between the plate vibrations. Therefore, acceleration sensors are installed on both plates to measure this phase difference. To estimate the internal acoustic characteristics, the sound pressure level in the cavity is measured using condenser microphones with a probe tube. The tips of the probe tubes are located near the plates and the cylinder wall, which are the approximate locations of the maximum sound pressure level when the sound field becomes resonant.

To perform the electricity generation experiment, the piezoelectric element is used and is comprised of the piezoelectric part constructed of ceramics and the electrode part constructed of brass, which have the diameters of 25 and $35 \mathrm{~mm}$ and the thicknesses of 0.23 and $0.30 \mathrm{~mm}$, respectively. Actually, the piezoelectric elements are installed at each center of both plates, as shown in Figures 2(a) and 2(b). The electric power generated by the expansion and contraction of the piezoelectric elements is discharged through the resistance circuit, which consists of three resistors having resistances $R_{v}, R_{i}$, and $R_{c}$, as shown in Figure 2(c). $R_{v}$ and $R_{i}$ are the resistances of the voltmeter and ammeter built-in the wattmeter and are $2 \mathrm{M} \Omega$ and $2 \mathrm{~m} \Omega$, respectively, while $R_{c}$ is the resistance of the resistor connected outside the wattmeter and is $97.5 \mathrm{k} \Omega$. To grasp the effect of vibroacoustic coupling on energy harvesting, the electric power is measured with and without the cylinder and is estimated by the comparison of both cases. In such a estimation, the electric power is normalized by the vibration power supplied with the plate, which is obtained from the point force and flexural displacement.

\section{Results and Discussion}

4.1. Acoustic Characteristics under Vibroacoustic Coupling. In the theoretical study, the plates are assumed to be aluminum having a Young's modulus $E$ of $71 \mathrm{GPa}$ and a Poisson's ratio $v$ of 0.33 . The radius $a$ and thickness $h$ of the plates are constant at $153 \mathrm{~mm}$ and $3 \mathrm{~mm}$, respectively, whereas the length of the cylindrical sound field having the same radius as that of the plates varies from 100 to $2000 \mathrm{~mm}$. The support conditions of the plates, which have flexural rigidity $D\left[=E h^{3} /\left\{12\left(1-\nu^{2}\right)\right\}\right]$, are expressed by the nondimensional stiffness parameters $T_{n}\left(=T_{1} a^{3} / D=T_{2} a^{3} / D\right)$ and $R_{n}\left(=R_{1} a / D=R_{2} a / D\right)$. These values are identical for both plates. If $R_{n}$ ranges from 0 to $10^{8}$ when $T_{n}$ is $10^{8}$, the support condition can be assumed from a simple support to a clamped support. The actual condition adopts $T_{n}=10^{8}$ and $R_{n}=10^{1}$ to get closer to the experimental support condition. These plates 1 and 2 are subjected to the point forces $F_{1}$ and $F_{2}$, which are set to $1 \mathrm{~N}$ and are located at $r_{1} / a=r_{2} / a=0.4$, respectively, as well as the actual excitation experiment. In particular, the analysis in which only one end plate is excited is carried out with taking $F_{2}$ as $0 \mathrm{~N}$.

The plate and sound field eigenfrequency characteristics involved in the vibroacoustic coupling are represented by the natural frequency $f_{n m}$ corresponding to the $(n, m)$ mode and the resonance frequency $f_{n p q}$ corresponding to the $(n, p, q)$

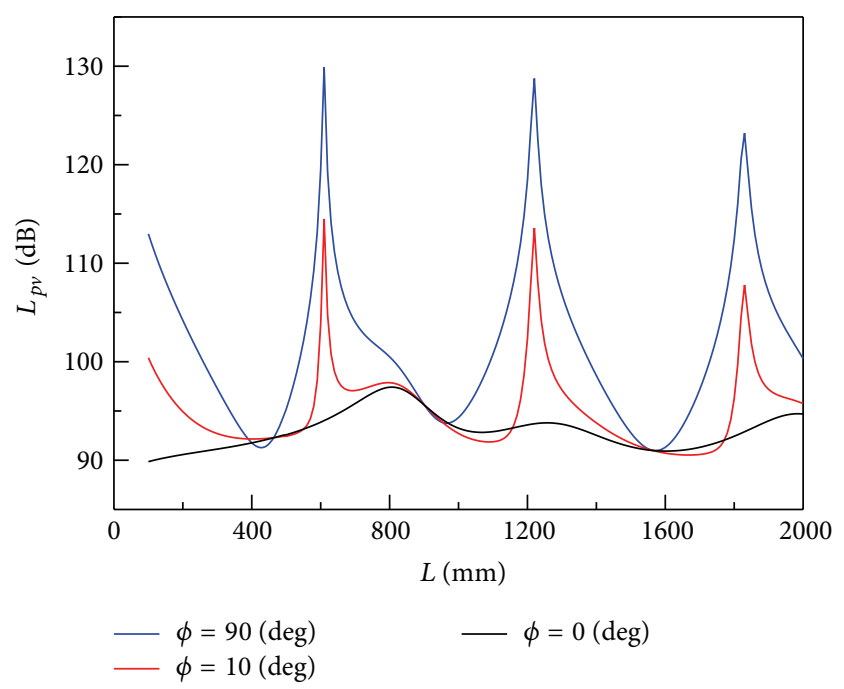

FIGURE 3: Variation in sound pressure level with cylinder length when phase difference changes.

mode. The excitation frequency is chosen as $f_{00}$ that makes the plates vibrate in the $(0,0)$ mode. With respect to the plate vibration, although the phase $\phi_{1}$ of plate 1 is fixed at $0 \mathrm{deg}$, the phase $\phi_{2}$ of plate 2 ranges from 0 to $180 \mathrm{deg}$, and then they are related by the phase difference $\phi$ as follows:

$$
\phi=\phi_{2}-\phi_{1} \text {. }
$$

Figure 3 shows the variations in $L_{p v}$ with $L$, when only plate 1 is excited and $\phi$ is arbitrarily set to 0,10 , and $90 \mathrm{deg}$. $L_{p v}$ varies only slightly over the entire range of $L$ when $\phi=0 \mathrm{deg}$, but varies substantially and exhibits peaks near $L=610,1220$, and $1830 \mathrm{~mm}$ when $\phi=10$ and $90 \mathrm{deg}$. The value of $L_{p v}$ is lower when $\phi=10$ and 90 deg and is almost identical at all phase differences near $L=460,920$ and $1560 \mathrm{~mm}$. To estimate the influence of each acoustic mode on these sound fields, the contribution $C_{E}$ can be defined as the ratio of the acoustic energy $E_{n p q}$ stored in the specific $(n, p, q)$ mode to the total acoustic energy $E_{\text {all }}$ of the entire sound field:

$$
C_{E}=\frac{E_{n p q}}{E_{\text {all }}} .
$$

Figures 4(a) and 4(b) show $C_{E}$ as a function of $L$ for the $(0,0, q)$ modes for values of $q$ between 0 and 4 for $\phi=0$ and $90 \mathrm{deg}$, respectively. In case of $\phi=0 \mathrm{deg}, C_{E}$ is relatively large when $L$ is short. The value of $C_{E}$ for the $(0,0,0)$ mode decreases as $L$ increases because of the increase in the value of $C_{E}$ for the $(0,0,1)$ mode. The $(0,0,0)$ mode is regarded as a pumping mode that corresponds to the reciprocation of both end plates supported by the aerostatic stiffness of the cavity. In the range of longer length, as the value of $q$ increases, $C_{E}$ decreases relatively to $C_{E}$ of the $(0,0,0)$ mode because the sound field is affected by other modes. At $\phi=$ $90 \mathrm{deg}, C_{E}$ varies dramatically when the acoustic mode that dominates the sound field changes, so that the range of $L$ over which the influence of such a dominant mode extends can be clearly distinguished. The acoustic mode $(0,0, q)$ causes $L_{p v}$ to have peaks at $L=610,1220$, and $1830 \mathrm{~mm}$, having 


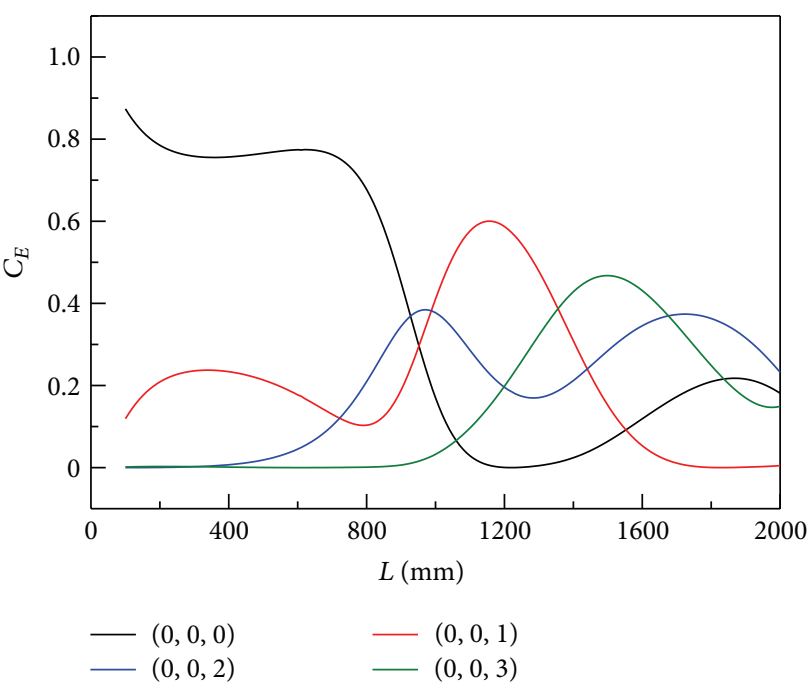

(a)

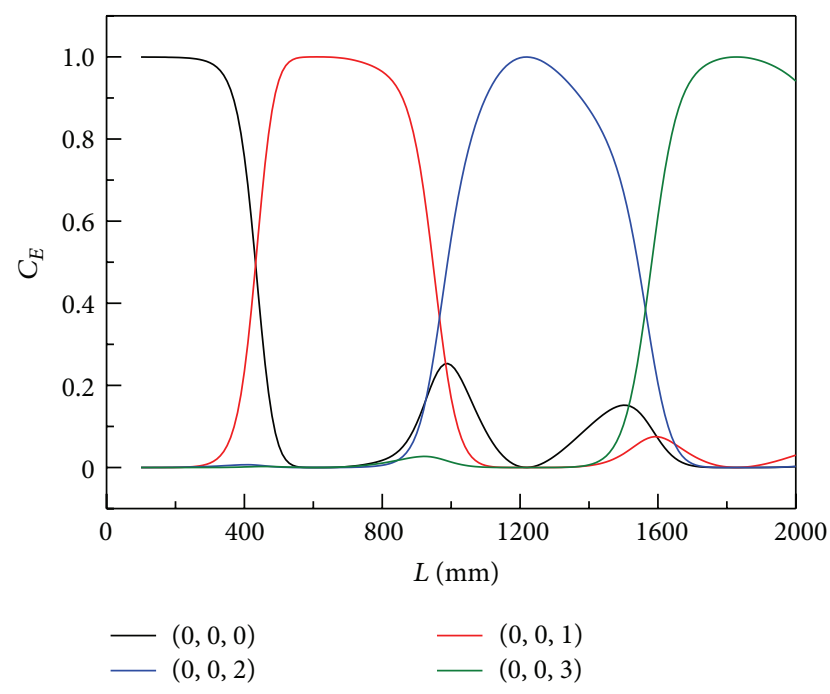

(b)

FIGURE 4: Contribution of acoustic mode to sound field at (a) $\phi=0$ [deg] and (b) $\phi=90$ [deg].

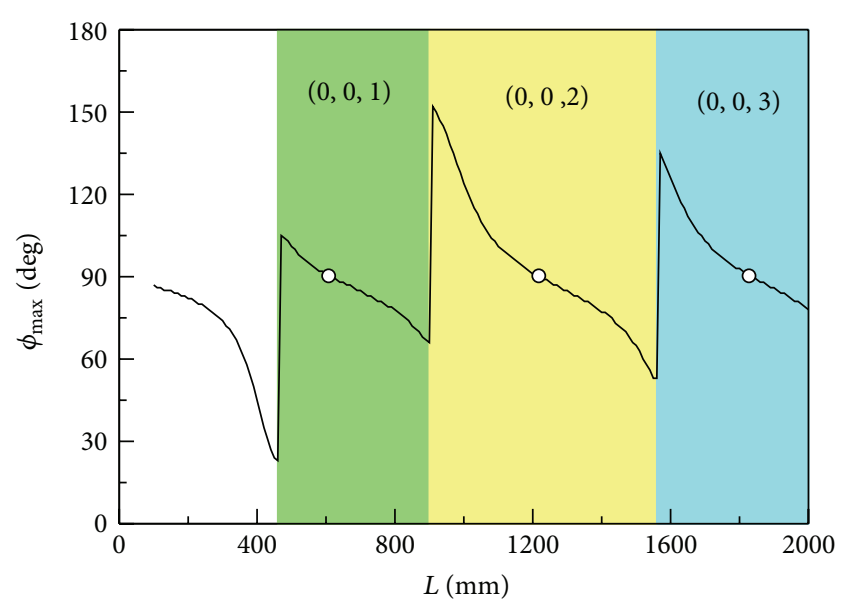

FIGURE 5: Variations in phase difference with cylinder length.

modal shapes similar to that of the $(0,0)$ mode of the plate vibrations. These peaks occur at an integer $q$ starting from $q=$ 1 with increasing $L$. The vibration of plate 2 has a significant effect on the formation of the sound field and vibroacoustic coupling, despite not being driven by the point force, whereas the influence of $\phi$ on the acoustic characteristics has only been described for $\phi=0,10$, and $90 \mathrm{deg}$. This change in $L_{p v}$ with $\phi$ indicates that the acoustic characteristics depend strongly on the vibration of plate 2 ; that is, there are ranges of $\phi$ that intensify or suppress coupling between the plate vibrations and sound field. Here the values of $\phi$ at which $L_{p v}$ is maximum are denoted by $\phi_{\max }$.

In Figure 5, the variations in $\phi_{\max }$ with $L$ and the values of $\phi_{\max }$ that maximize $L_{p v}$ are plotted by a line and circles, respectively. $\phi_{\max }$ is approximately $87 \mathrm{deg}$ at $L=100 \mathrm{~mm}$ and decreases gradually with increasing $L$ up to approximately $L=460 \mathrm{~mm}$, where $\phi_{\max }$ suddenly increases to over $90 \mathrm{deg}$ and then decreases again with increasing $L$. This behavior of $\phi_{\max }$ is repeated in a similar manner as $L$ increases to $L=2000 \mathrm{~mm}$. Peaks in $L_{p v}$ indicate that vibroacoustic coupling between the plate vibrations and sound field is promoted at approximately $90 \mathrm{deg}$. $f_{n m}$ and $f_{n p q}$ must be approximately equal for the promotion of this coupling, so that the acoustic modes involved in vibroacoustic coupling are greatly influential around the lengths at which $L_{p v}$ peaks. On the other hand, we confirm that the longitudinal order $q$ shifts from 1 to 2 and from 2 to 3 at $L=900$ and $1560 \mathrm{~mm}$ where $L_{p v}$ varies abruptly, respectively, based on the distributions of the sound pressure level along the $z$ direction inside the cavity. As a result, the sound fields, which are classified in the ranges of 470 to $900 \mathrm{~mm}, 910$ to $1560 \mathrm{~mm}$, and 1570 to $2000 \mathrm{~mm}$, are dominated by the $(0,0,1),(0,0,2)$, and $(0,0,3)$ modes, respectively, as shown in this figure where the range of the acoustic mode is classified in color. Naturally, this classification is also thought to be due to variations in the dominant acoustic mode, whose contribution $C_{E}$ is maximized in Figure 4(b).

Figure 6 shows the sound pressure levels $L_{p 1}$ and $L_{p 2}$, which are measured near plates 1 and 2 , respectively, as functions of $L$. The theoretical level $L_{p v}$, which is maximized at each $L$ when the phase difference $\phi$ ranges from 0 to $180 \mathrm{deg}$, is also indicated to compare with the experimental results. $L_{p 1}$ and $L_{p 2}$ show peaks around 615, 1275, and $1900 \mathrm{~mm}$, and these levels are almost coincident for each peak. However, they decrease in the middle range of those lengths. In particular, decreases in $L_{p 1}$ are remarkable and their differences expand around 950 and $1550 \mathrm{~mm} . L_{p v}$ also shows peaks at 610,1230, and $1840 \mathrm{~mm}$ and corresponds approximately with the above lengths where $L_{p 1}$ and $L_{p 2}$ peak.

Since the above results are derived from the investigation based on the excitation of one end plate, furthermore, the excitation condition in which both plates are subjected to the same excitation force is taken to grasp the effect of the excitation method on vibroacoustic coupling. Figure 7 shows 


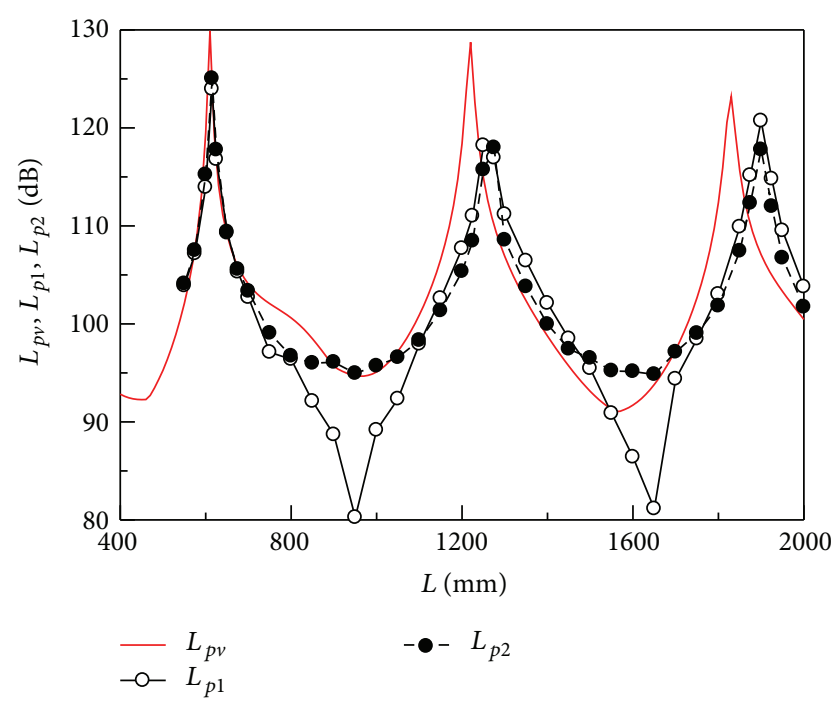

FIGURE 6: Comparison between theoretical and experimental results for sound pressure level.

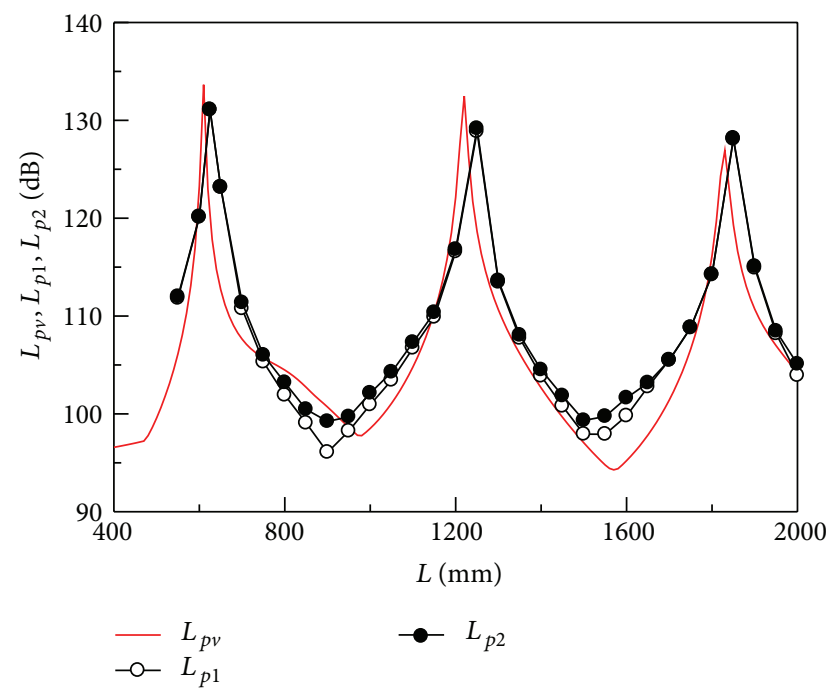

FIGURE 7: Comparison between theoretical and experimental results for sound pressure level when both end plates are excited by point force.

the variations in $L_{p v}$ corresponding to $\phi_{\max }$ and the variations in $L_{p 1}$ and $L_{p 2}$ that are measured in the experiment and are maximized when the phase difference between both point forces ranges from $0 \mathrm{deg}$ to $180 \mathrm{deg}$. Peaks in $L_{p v}$ appear at $L=$ 610,1230 , and $1840 \mathrm{~mm}$. These peaks are known to be caused by the $(0,0,1),(0,0,2)$, and $(0,0,3)$ modes, respectively. Note that $L_{p 1}$ and $L_{p 2}$ increase greatly at 625,1250 , and $1850 \mathrm{~mm}$. However, $L_{p 1}$ and $L_{p 2}$ are hardly distinguished in the middle range of lengths where the sound pressure levels peak, having been different in the results for the excitation of one end plate, as shown in Figure 6.

4.2. Plate Vibration Characteristics under Vibroacoustic Coupling. Since the plate vibrations influence the acoustic characteristics via vibroacoustic coupling, the magnitude of the

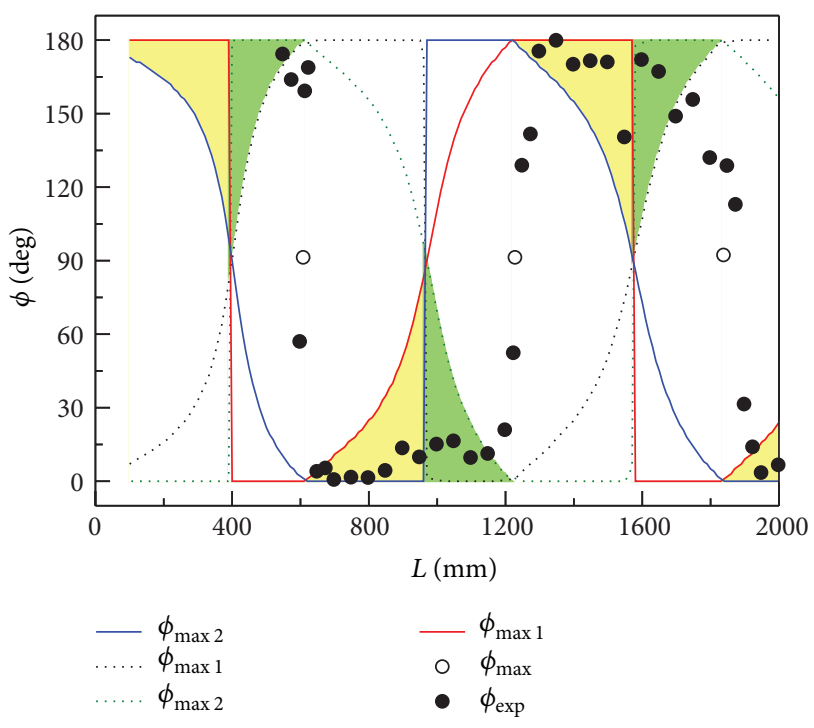

FIGURE 8: Comparison between theoretical and experimental results for phase difference.

flexural displacements $w_{1}$ and $w_{2}$ is also significant in studying the effect of the plate vibrations on the sound field. Here the phase differences are denoted as $\phi_{\max 1}$ and $\phi_{\max 2}$ when $w_{1}$ and $w_{2}$ are maximized, while they are denoted as $\phi_{\min 1}$ and $\phi_{\min 2}$ when $w_{1}$ and $w_{2}$ are minimized. Figure 8 shows $\phi_{\max 1}, \phi_{\max 2}, \phi_{\min 1}$, and $\phi_{\min 2}$ as functions of $L$ when only plate 1 is excited. $\phi_{\max 1}$ is constant at $180 \mathrm{deg}$ for $L$ ranging from 100 to $390 \mathrm{~mm}$ and decreases abruptly up to $0 \mathrm{deg}$ at $L=400 \mathrm{~mm}$. Then, remaining constant at $0 \mathrm{deg}$ up to $L=610 \mathrm{~mm}, \phi_{\max 1}$ increases gradually with $L$ and returns to $180 \mathrm{deg}$ at $L=1220 \mathrm{~mm}$, increasing somewhat abruptly near $L=970 \mathrm{~mm}$. Beyond $L=1220 \mathrm{~mm}, \phi_{\max 1}$ is again constant at $180 \mathrm{deg}$ up to $L=1570 \mathrm{~mm}$, and this behavior is repeated as $L$ increases to $L=2000 \mathrm{~mm}$. $\phi_{\max 2}$ exhibits gradual and abrupt changes similar but alternate to $\phi_{\max 1}$. For example, when $L$ increases, a gradual decrease occurs in $\phi_{\max 2}$ between $L=100$ and $620 \mathrm{~mm}$, and an abrupt increase occurs near $L=970 \mathrm{~mm}$. Both $\phi_{\max 1}$ and $\phi_{\max 2}$ shift between 0 and 180 deg with changing $L$ and intersect at approximately 90 deg and near the length at which $L_{p v}$ peaked in Figure 6. $\phi_{\min 1}$ and $\phi_{\min 2}$ behave exactly alike but opposite to $\phi_{\max 1}$ and $\phi_{\max 2}$.

In Figure 8, the theoretical results for $\phi_{\max }$ where $L_{p v}$ peaks and the experimental results for $\phi_{\exp }$ as $L_{p 1}$ and $L_{p 2}$ are maximized at each $L$ are also plotted. $\phi_{\text {exp }}$ ranges greatly between in-phase and out-of-phase and $\phi_{\max }$ exists in the process where $\phi_{\exp }$ changes abruptly. Then, $\phi_{\exp }$ lies in the light yellow areas surrounded by $\phi_{\max 1}$ and $\phi_{\max 2}$ in the $L$ ranges longer than the lengths when the sound pressure level peaks and occurs in the yellowish green areas surrounded by $\phi_{\min 1}$ and $\phi_{\min 2}$ on the other side. In other words, since vibroacoustic coupling is gradually weakened with increasing $L$ after the peaks of $L_{p 1}$ and $L_{p 2}$, the acoustic mode involved in coupling shifts to that having the next order $q$.

Figure 9 shows the vibration levels $L_{v 1}$ and $L_{v 2}$ of plates 1 and 2 as functions of $L$ and the accelerations $\alpha_{1}$ and $\alpha_{2}$ of 


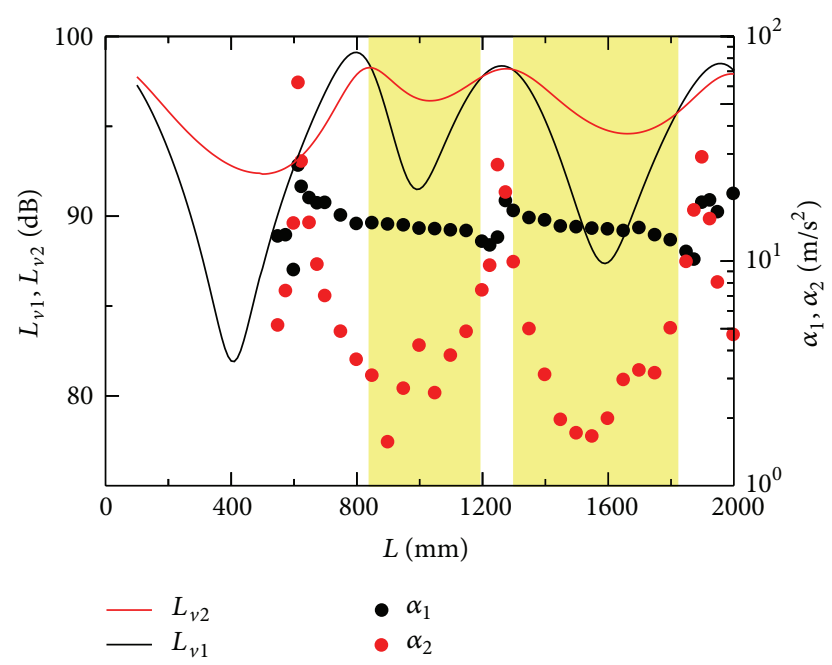

FIGURE 9: Comparison between characteristics of theoretical vibration and experimental acceleration.

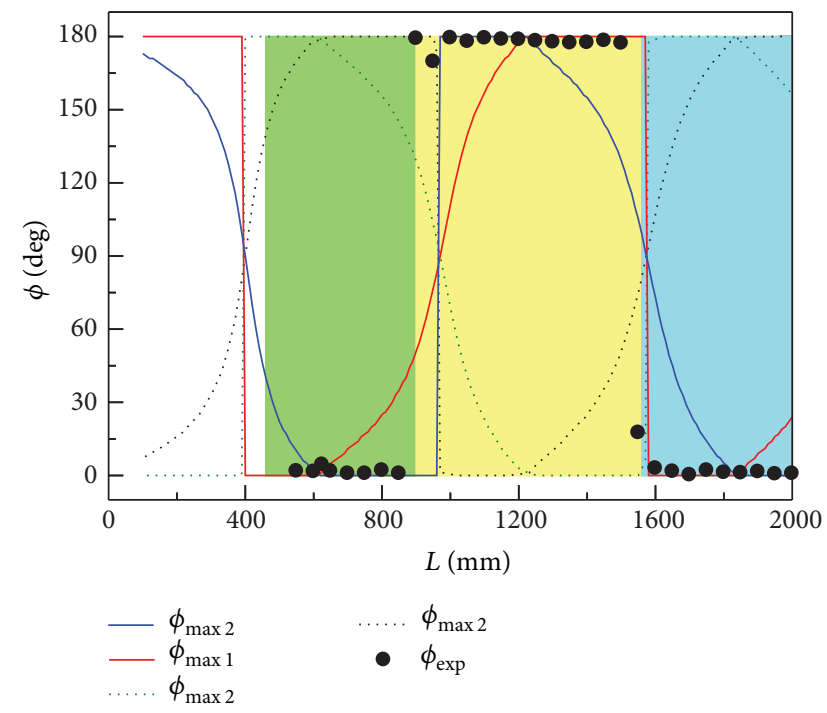

Figure 10: Comparison between theoretical and experimental results for phase difference when both end plates are excited by point force.

plates 1 and 2 are also plotted to compare with the theoretical plate behavior. $L_{v 1}$ is smaller than $L_{v 2}$ in the ranges of 100 to $610 \mathrm{~mm}, 800$ to $1200 \mathrm{~mm}$, and 1300 to $1820 \mathrm{~mm}$, so that $L_{v 1}$ and $L_{v 2}$ intersect at a number of $L$ and the intersections take place around the lengths where $L_{p v}$ peaks. The actual motion of plate 1 is almost suppressed by that of the vibrator since plate 1 is supported by the vibrator; hence, $\alpha_{1}$ is approximately constant over the entire $L$ range. However, since the motion of plate 2 depends greatly on the behavior of the sound field, that is, the only excitation source for plate $2, \alpha_{2}$ peaks at $L=650,1280$, and $1880 \mathrm{~mm}$ and is suppressed in the other ranges of $L$, such as variations in $L_{p 1}$ and $L_{p 2}$. As a result, the relative relationship of $\alpha_{1}$ and $\alpha_{2}$ becomes opposite to that of $L_{v 1}$ and $L_{v 2}$ as shown in the colored regions. In the theoretical analysis, we confirm that the distributions of the sound pressure level along the $z$ direction inside the cavity behave in a similar manner to the sound field inside a sound tube having single closed and open ends at the lengths, around which the sound pressure level decreased in Figure 6. These distributions occur in the process of shifting acoustic modes because of changing the cylinder length and have the opposite tendency for the difference between $L_{p 1}$ and $L_{p 2}$ in those middle ranges of lengths where the sound pressure levels peaked in Figure 6. This is derived from the difference between the tendencies of the above vibration level and acceleration with respect to both plates. If the experimental model completely emulated the theoretical model in the flexural displacement, such a discrepancy would not take place.

In Figure 10, the experimental phase difference $\phi_{\text {exp }}$, at which $L_{p 1}$ and $L_{p 2}$ are maximized, is compared with the theoretical phase differences $\phi_{\max 1}, \phi_{\max 2}, \phi_{\min 1}$, and $\phi_{\min 2}$ when both plates are subjected to the same excitation force, as shown in Figure 8 . The shifts in the $(0,0, q)$ modes are also represented by the changing colors. $\phi_{\text {exp }}$ shifts between 0 and 180 deg with changing $L$ and corresponds approximately with the $\phi_{\max 1}$ and $\phi_{\max 2}$ that behave uniformly in the vicinity of the in-phase side or the out-of-phase side. In this way, the behavior of the phase difference is very different in the excitation method.

4.3. Electricity Generation Characteristics. In this section, we consider electricity generation by the plate vibrations coupled with the sound field. In this case, the electricity generation is estimated by the comparison between the electric power via the piezoelectric element and the mechanical power supplied to the plate by the vibrator that is obtained from the relationship between the point force and flexural displacement at the excitation point. Figure 11 shows the relationship between the electric power $P_{e}$ and mechanical power $P_{m}$ when the point force $F_{1}$ ranges from 1 to $5 \mathrm{~N}$. In this case, the cylinder is removed; the plate vibrations do not couple with the internal sound field. Although $P_{e}$ is considerably smaller than $P_{m}$, their relationship is directly proportional. Here, the relationship between $P_{e}$ and $P_{m}$ is defined as

$$
P_{e m}=\frac{P_{e}}{P_{m}} .
$$

Figure 12 shows variations in $P_{e m}$ with $L$; the powers are measured in the experimental apparatus via the cylinder shown in Figure 2(a) when one end plate is excited by the point force. Although $P_{e m}$ of plate 1 remains almost constant over the entire range of $L, P_{e m}$ of plate 2 increases greatly at $L=615,1275$, and $1900 \mathrm{~mm}$. It is natural that the relationship between $P_{e m}$ of plate 1 and 2 is derived from the behavior of $\alpha_{1}$ and $\alpha_{2}$ in Figure 9. $P_{e m}$ is considered as energy-harvesting efficiency. In an electricity generation by means of beam or plate vibrations with piezoelectric elements, coupling between the structural vibration and electric field, that is, electromechanical coupling should be considered to relate the in-plane stress to the applied electric field. Considering that the behaviors of $P_{e m}$ for plates 1 and 2 correspond to those of 
$\alpha_{1}$ and $\alpha_{2}$ and the values of $P_{e m}$ are substantially small, that is, the value of $P_{e}$ is extremely small in comparison with that of $P_{m}$, we assume that the behavior of plate vibrations is hardly affected by electromechanical coupling in this study.

Moreover, to grasp the effect of vibroacoustic coupling on electricity generation, although $P_{R}$ is taken as the ratio of $P_{e m}$ measured with and without the cylinder, $P_{e m}$ measured with the cylinder is obtained from the total value $P_{e}$ with respect to plates 1 and 2. Figure 13 shows variations in $P_{R}$ with $L$; the results of the excitation of both ends are also indicated to study the effect of the excitation method. $P_{R}$ for the excitation of one end is maximized as the sound pressure level peaks due to the promotion of vibroacoustic coupling. This is because the plate vibration on the nonexcitation side contributes strongly to the electricity generation, whereas their $L$ ranges are limited to the narrow regions in comparison with the sound pressure level. Since the contribution of the nonexcitation side is extremely weakened in other ranges of the above $L$, the effect of the excitation side is uniformly maintained, as shown in Figure 12. On the other hand, at the excitation of both ends, there is not such a nonexcitation side that contributes greatly to the electricity generation, so that $P_{R}$ never reaches values as large as those of the excitation of one end, when the sound pressure level is maximized. In $L$ ranges where vibroacoustic coupling is weakened, $P_{R}$ remains almost constant and is close to that of the excitation of one end. Under the situation of such a weakened vibroacoustic coupling, the excitation of both ends becomes approximately twice as large as the excitation of one end in the respective total amounts of the electric power $P_{e}$ and mechanical power $P_{m}$. As a result, $P_{R}$ shifts almost constantly with $L$, no matter what the excitation method is.

Hence, it follows that the excitation of one end is decidedly superior to that of both ends by electricity generation efficiency, according to this estimation method. These results are remarkable in the viewpoint where the acoustic energy from the sound radiation can be harvested through vibroacoustic coupling. However, the number or area of the piezoelectric element should be increased to improve the efficiency $P_{e m}$, so that the effect of electromechanical coupling on the plate vibration must be taken into consideration in the theoretical procedure.

\section{Conclusion}

To apply vibroacoustic coupling to electricity generation, as a means of harvesting energy from vibration systems, coupling between plate vibrations and a sound field was investigated theoretically and experimentally for a cylindrical structure with thin circular end plates. The end plate was subjected to a harmonic point force. Moreover, the effect of vibroacoustic coupling on the harvest of energy was estimated from the electricity generating experiment. The present study focused on promoting the vibroacoustic coupling to increase the flexural displacements of the plates and the sound pressure level inside the cavity.

As a result of the estimation of vibroacoustic coupling from various viewpoints, the theoretical study reveals that

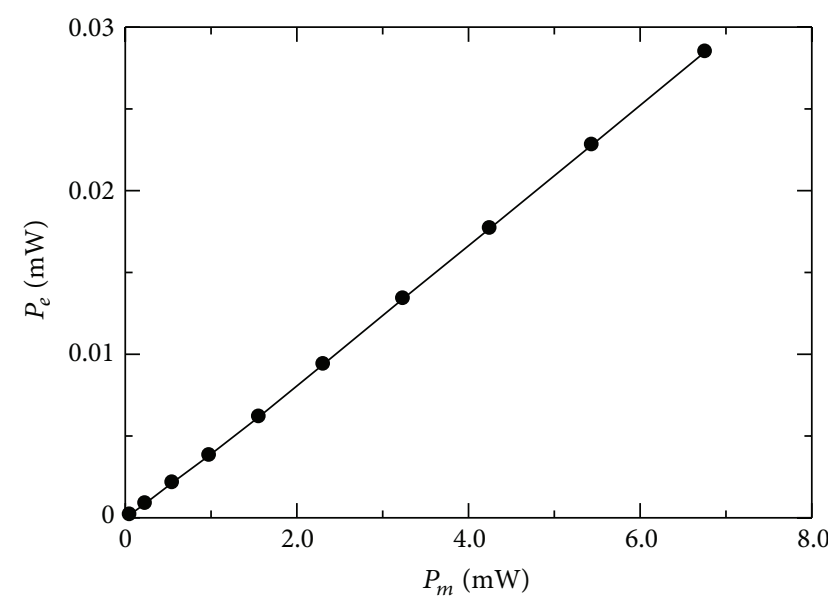

FIGURE 11: Relationship of electric power and mechanical power.

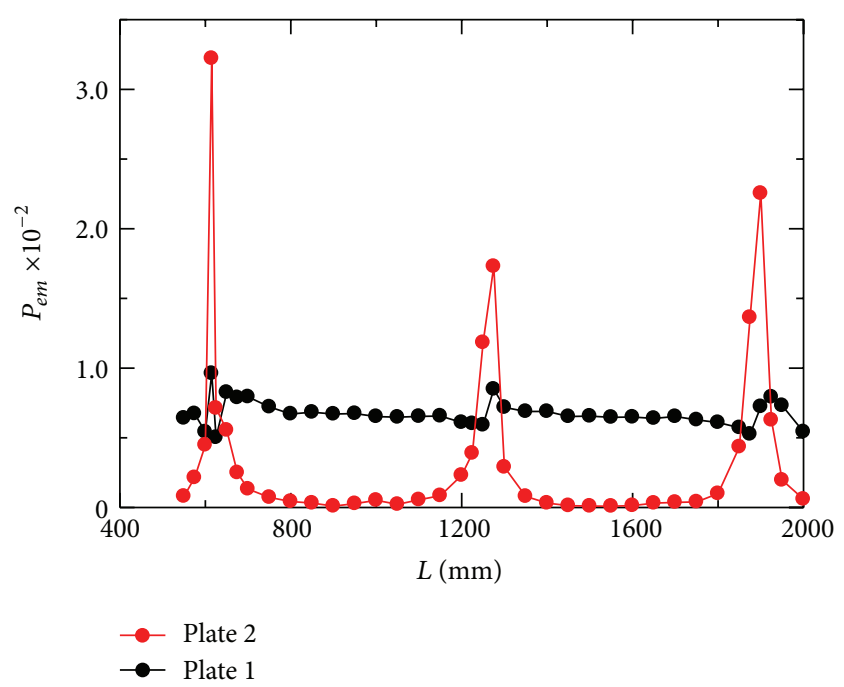

FIGURE 12: Energy-harvesting efficiency as function of cylinder length.

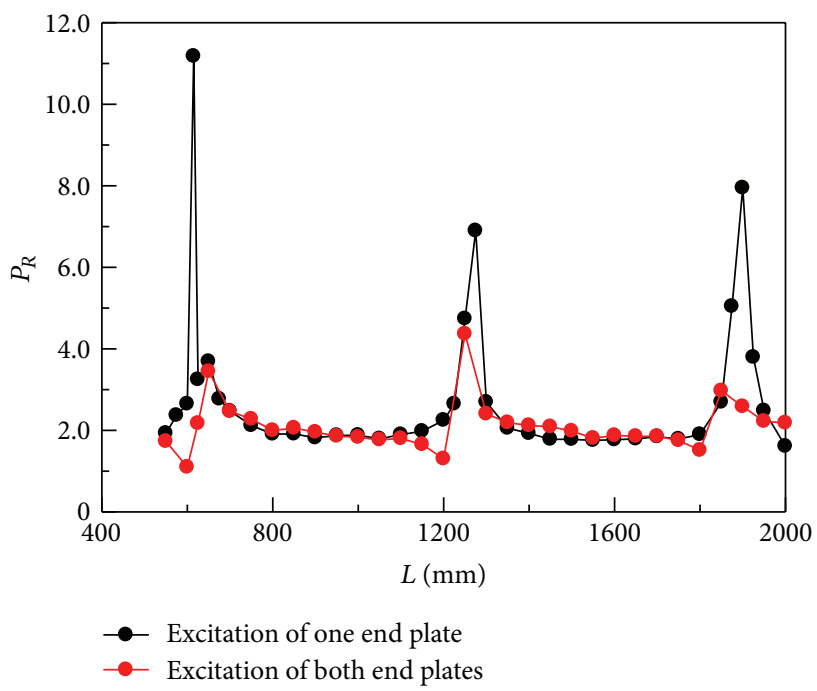

FIGURE 13: Effect of vibroacoustic coupling on energy harvesting for excitations of one end plate and both end plates. 
the closeness of eigenfrequencies and the similarity of modal shapes between the plate vibrations and sound field are indispensable for promoting coupling. The experimental results confirm that the theoretical estimation of increasing the flexural displacement and sound pressure level via the promotion of vibroacoustic coupling support the complicated acoustic characteristics deduced from the theoretical results. In particular, changes in the cylinder length shift the acoustic mode in the longitudinal order and vary periodically the phase difference between both plate vibrations. It is validated that the phase difference is greatly different in the excitation method. When vibroacoustic coupling is promoted, the electricity generation experiment verifies that the promotion of coupling causes the generation efficiency to improve in comparison with the electricity generation caused only by the plate vibration without coupling.

\section{References}

[1] S. R. Anton and H. A. Sodano, "A review of power harvesting using piezoelectric materials (2003-2006)," Smart Materials and Structures, vol. 16, no. 3, pp. 1-21, 2007.

[2] K. H. Mak, S. McWilliam, A. A. Popov, and C. H. J. Fox, "Performance of a cantilever piezoelectric energy harvester impacting a bump stop," Journal of Sound and Vibration, vol. 330, no. 25, pp. 6184-6202, 2011.

[3] S. Backhaus and G. W. Swift, "A thermoacoustic stirling heat engine," Nature, vol. 399, no. 6734, pp. 336-338, 1999.

[4] S. Sakamoto, D. Tsukamoto, Y. Kitadani, T. Ishino, and Y. Watanabe, "Effect of sub-loop tube on energy conversion efficiency of loop-tube-type thermoacoustic system," in Proceedings of the 20th International Congress on Acoustics (ICA '10), pp. 13611362, 2010 (Japanese).

[5] K. Hayamizu, "Device for electric generation," Japanese patent disclosure, 2010-200607, 2010 (Japanese).

[6] K. Hayamizu, R. Ando, and Y. Takefuji, "Simultaneous providing device of baseband and carrier signal using sound-generated electricity," Mobile Multimedia Communications, vol. 105, no. 80, pp. 47-49, 2005 (Japanese).

[7] J. Pan and D. A. Bies, "The effect of fluid-structural coupling on sound waves in an enclosure-theoretical part," Journal of the Acoustical Society of America, vol. 87, no. 2, pp. 691-707, 1990.

[8] J. Pan and D. A. Bies, "The effect of fluid-structural coupling on sound waves in an enclosure-experimental part," Journal of the Acoustical Society of America, vol. 87, no. 2, pp. 708-717, 1990.

[9] L. Cheng and J. Nicolas, "Radiation of sound into a cylindrical enclosure from a point-driven end plate with general boundary conditions," Journal of the Acoustical Society of America, vol. 91, no. 3, pp. 1504-1513, 1992.

[10] L. Cheng, "Fluid-structural coupling of a plate-ended cylindrical shell: vibration and internal sound field," Journal of Sound and Vibration, vol. 174, no. 5, pp. 641-654, 1994.

[11] H. Moriyama, "Acoustic characteristics of sound field in cylindrical enclosure with exciting end plates," Transactions of Japan Society of Mechanical Engineers C, vol. 69, no. 679, pp. 603-610, 2003 (Japanese).

[12] H. Moriyama and Y. Tabei, "Acoustic characteristics inside cylindrical structure with end plates excited at different frequencies," Journal of Visualization, vol. 7, no. 1, pp. 93-101, 2004.

[13] H. Moriyama, Y. Tabei, and N. Masuda, "Acoustic characteristics of a sound field inside a cylindrical structure with excited end plates: influence of excitation position on vibro-acoustic coupling," Acoustical Science and Technology, vol. 26, no. 6, pp. 477-485, 2005.

[14] W. Larbi, J.-F. Deü, and R. Ohayon, "Finite element formulation of smart piezoelectric composite plates coupled with acoustic fluid," Composite Structures, vol. 94, no. 2, pp. 501-509, 2012.

[15] P. M. Morse and K. U. Ingard, Theoretical Acoustics, McGrawHill, New York, NY, USA, 1968. 

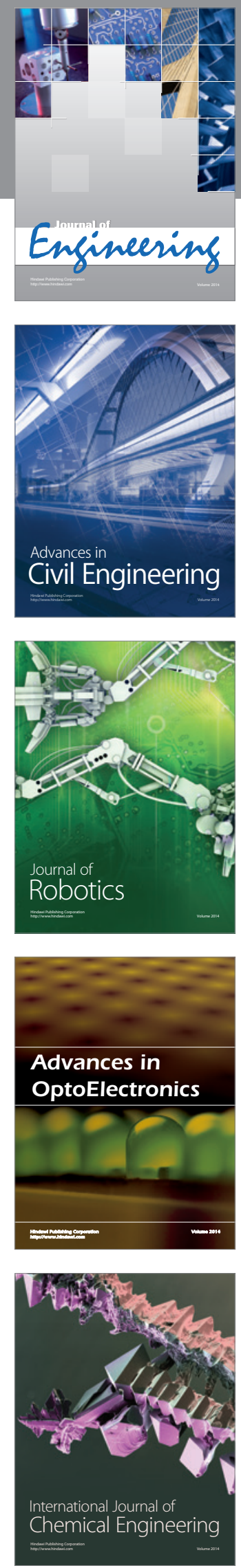

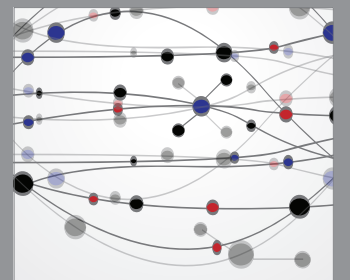

The Scientific World Journal
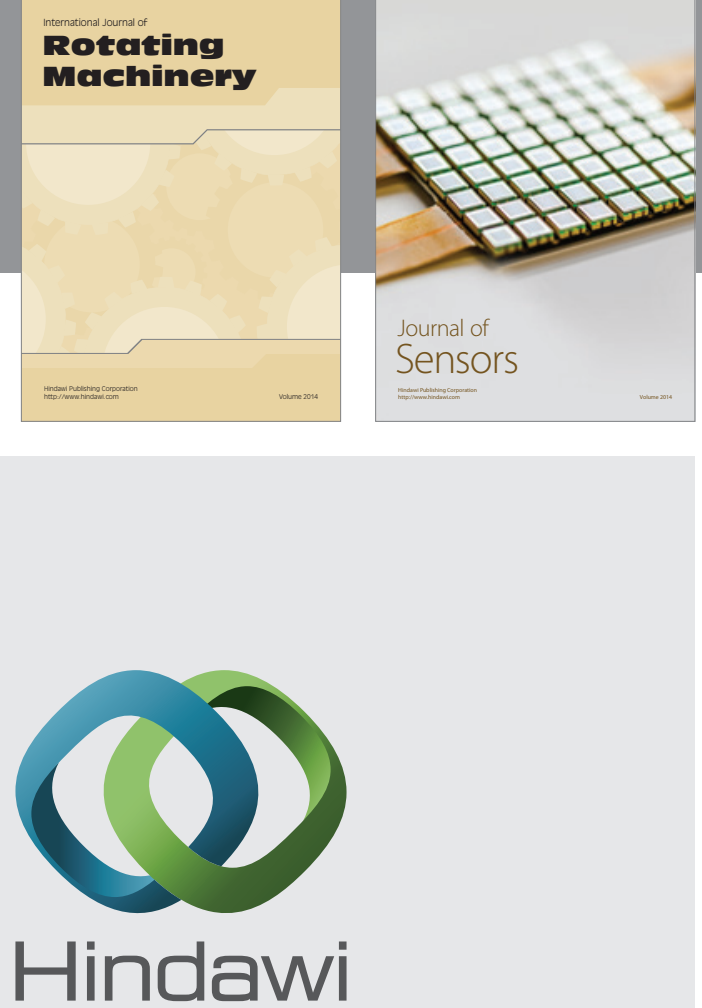

Submit your manuscripts at http://www.hindawi.com
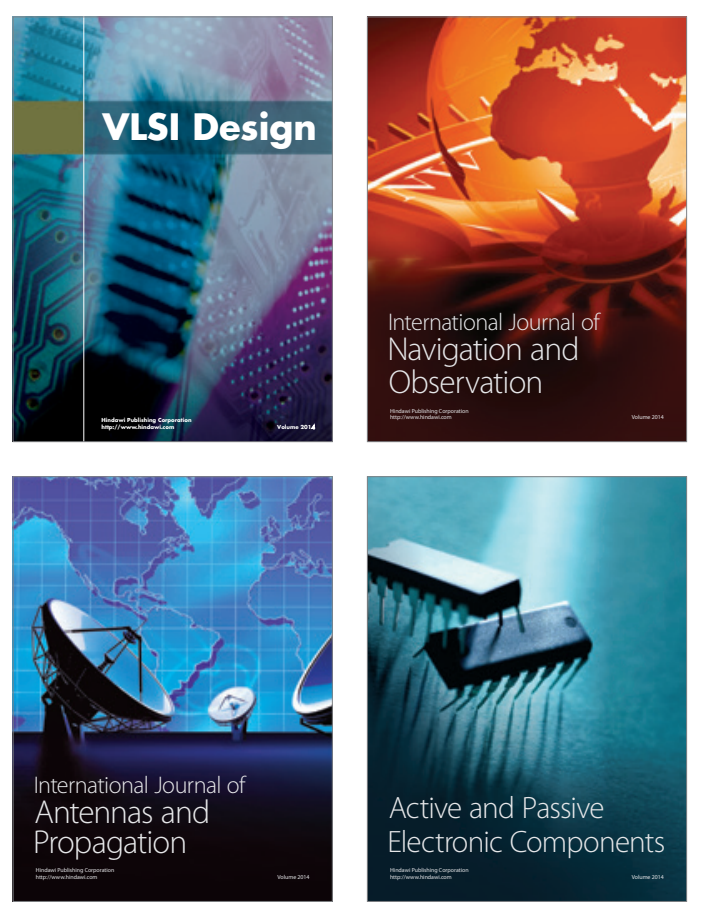
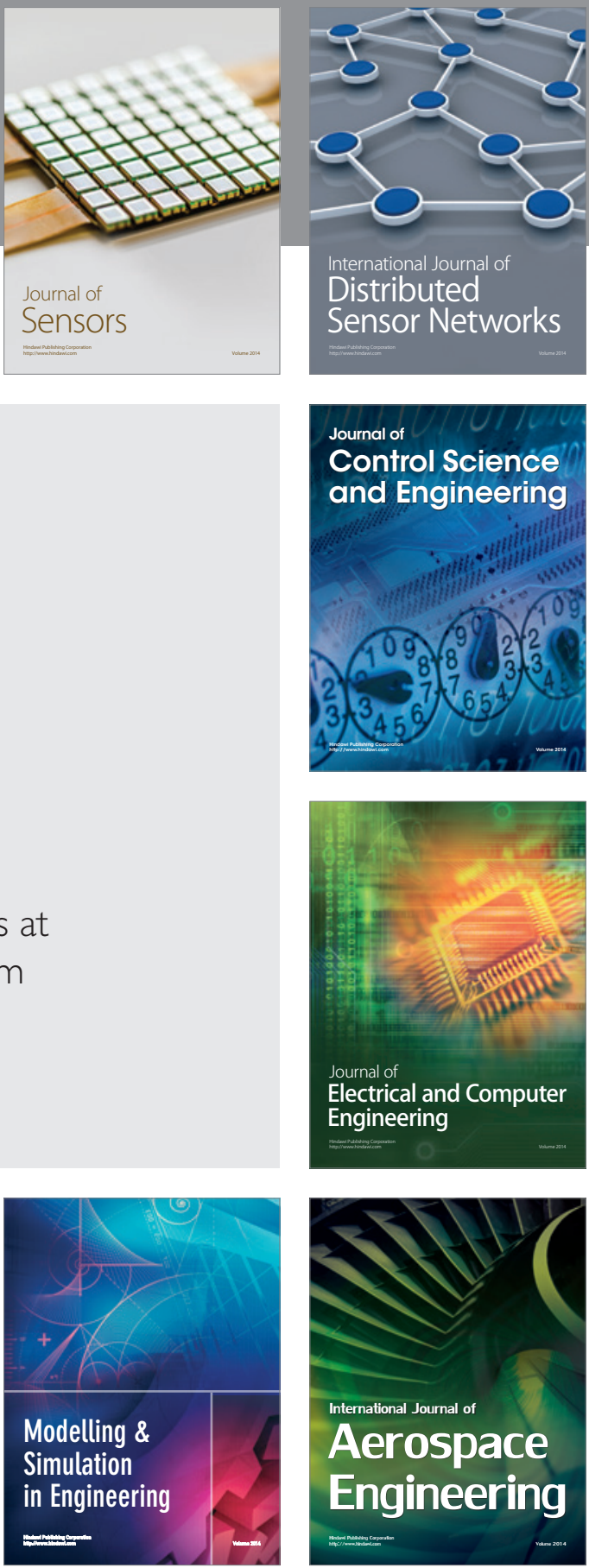

Journal of

Control Science

and Engineering
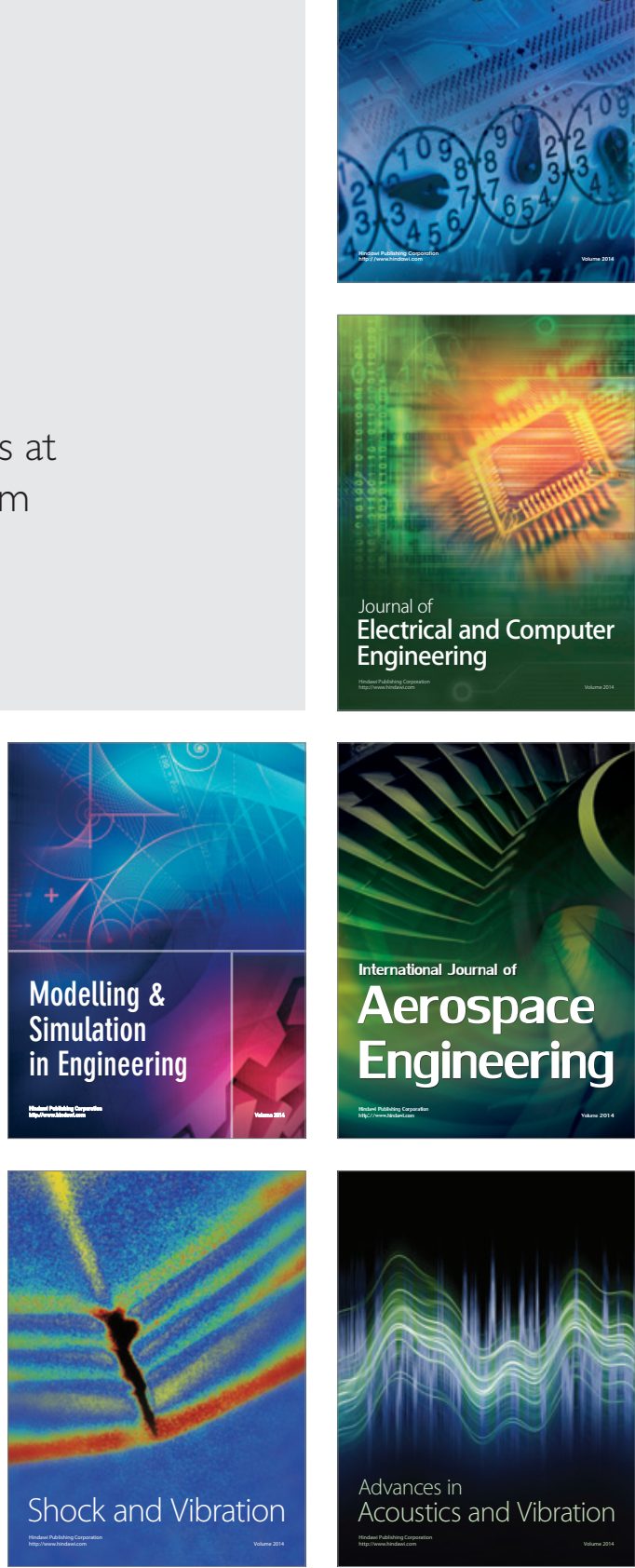